\title{
Improvement of Hemodynamic Parameters in Patients With Preserved Left Ventricular Systolic Function by Catheter Ablation of Atrial Fibrillation
}

- A Prospective Study Using Impedance Cardiography -

\author{
Yosuke Nakatani, MD, PhD; Tamotsu Sakamoto, MD, PhD; Yoshiaki Yamaguchi, MD, PhD; \\ Yasushi Tsujino, MD; Naoya Kataoka, MD, PhD; Kunihiro Nishida, MD, PhD; \\ Koichi Mizumaki, MD, PhD; Koichiro Kinugawa, MD, PhD
}

\begin{abstract}
Background: The effects of catheter ablation for atrial fibrillation (AF) on hemodynamic parameters in patients with preserved left ventricular (LV) systolic function are unclear.

Methods and Results: We enrolled 178 patients with AF (paroxysmal, 108; persistent, 70) with preserved LV systolic function who underwent AF ablation. The stroke volume index (SVI) was repeatedly measured using impedance cardiography. Reduced SVI (SVI, $<33 \mathrm{~mL} / \mathrm{m}^{2}$ ) was observed in $55 \%$ of patients before ablation. In patients with paroxysmal AF, the SVI did not change immediately after ablation (from $35 \pm 6 \mathrm{~mL} / \mathrm{m}^{2}$ to $35 \pm 5 \mathrm{~mL} / \mathrm{m}^{2} ; \mathrm{P}=0.652$ ); however, it increased 1 month after ablation and further increased $6 \mathrm{months}$ after ablation (1 month, $37 \pm 6 \mathrm{~mL} / \mathrm{m}^{2}, \mathrm{P}<0.001 ; 6$ months, $38 \pm 6 \mathrm{~mL} / \mathrm{m}^{2}, \mathrm{P}<0.001$ ). In patients with persistent $A F$, the $S V I$ increased immediately after ablation (from $30 \pm 5 \mathrm{~mL} / \mathrm{m}^{2}$ to $36 \pm 6 \mathrm{~mL} / \mathrm{m}^{2} ; \mathrm{P}<0.001$ ) and further increased until 6 months after ablation (1 month, $37 \pm 6 \mathrm{~mL}, \mathrm{P}<0.001 ; 6$ months, $\left.38 \pm 5 \mathrm{~mL} / \mathrm{m}^{2}, \mathrm{P}<0.001\right)$. The baseline $\mathrm{SVI}$ was the strongest predictor of the cardiac function improvement with an area under the curve of 0.828 .
\end{abstract}

Conclusions: The restoration and maintenance of sinus rhythm using catheter ablation increased the SVI in patients with preserved LV systolic function.

Key Words: Atrial fibrillation; Cardiac function; Catheter ablation; Impedance cardiography; Stroke volume

A trial fibrillation (AF), the most common sustained tachyarrhythmia observed in clinical practice, is related to potentially serious complications and death. ${ }^{1}$ AF causes loss of atrial contraction, decreased left ventricular (LV) diastolic filling, and resultant deterioration of cardiac function. In addition, tachycardia-induced cardiac dysfunction occurs in patients with AF with poor rate control. Consequently, patients with prevalent AF are reported to have a high cumulative incidence of heart failure, and AF occurrence is related to poor prognosis in patients with heart failure. ${ }^{2}$ Furthermore, a previous meta-analysis reported that the primary cause of death in patients with AF was cardiac. ${ }^{3}$

Some previous studies reported that catheter ablation for AF improved cardiac function in patients with $\mathrm{LV}$ systolic dysfunction..$^{47}$ However, the efficacy of AF ablation on the cardiac function of patients with the preserved LV systolic function remains unclear. Perhaps, potential cardiac dysfunction might exist even in patients with AF with preserved LV systolic function because atrial contractile dysfunction can be caused by AF. As cardiac function was assessed on the basis of the LV ejection fraction (LVEF) in the previous studies, ${ }^{4-7}$ the recovery of atrial contractile function might have been underestimated. Consequently, the improvement in cardiac function following AF ablation could have been underestimated.

The LV stroke volume (SV) is a vital hemodynamic parameter characterizing global cardiac function, including both left atrial and LV functions. However, it is not frequently used for following up cardiac function because precise measurement of SV has usually been performed invasively. Impedance cardiography (ICG) is a noninvasive method of evaluating SV based on measurement of the thoracic bioimpedance..$^{8-13}$ Being a noninvasive method, ICG can be performed repeatedly, even in the outpatient clinic setting. Furthermore, sequential measurements of

Received August 12, 2018; revised manuscript received September 16, 2018; accepted October 2, 2018; J-STAGE Advance Publication released online October 30, 2018 Time for primary review: 32 days

Second Department of Internal Medicine, University of Toyama, Toyama (Y.N., T.S., Y.Y., Y.T., N.K., K.K.); Nishida Medical Clinic, Toyama (K.N.); and Alpen Murotani Clinic, Toyama (K.M.), Japan

Mailing address: Yosuke Nakatani, MD, PhD, Second Department of Internal Medicine, University of Toyama, 2630 Sugitani, Toyama 930-0194, Japan. E-mail: yosuke3 gbst@gmail.com

ISSN-1346-9843 All rights are reserved to the Japanese Circulation Society. For permissions, please e-mail: cj@j-circ.or.jp 
SV are especially crucial for understanding the cardiac function improvement after AF ablation because considerable time is required for recovery from atrial contractile dysfunction and tachycardia-induced cardiac dysfunction. ${ }^{14,15}$

Thus, ICG might provide new insights into the efficacy of AF ablation for cardiac function. Hence, this study aimed to assess the changes over time in hemodynamic parameters, including SV, following AF ablation using ICG in patients with preserved LV systolic function.

\section{Methods}

\section{Study Population}

We prospectively enrolled consecutive patients with AF who underwent ablation at the Toyama University Hospital (Toyama, Japan) from April 2014 to March 2017. We excluded patients with previous catheter ablation, LV systolic dysfunction (LVEF $<50 \%$ ), prior heart surgery, hemodialysis, thyroid diseases, and pulmonary diseases. Paroxysmal AF was defined as AF lasting $<7$ days, persistent $\mathrm{AF}$ was defined as AF lasting $\geq 7$ days but $<1$ year, and longstanding persistent AF was defined as continuous AF lasting $\geq 1$ year. ${ }^{16}$ If AF recurred during the follow-up period, we excluded the patient from the study. Overall, we enrolled 194 patients, but 7 and 9 patients were excluded because of AF recurrence and loss to follow-up, respectively. Consequently, we evaluated 178 patients, comprising 108 with paroxysmal AF and 70 with persistent AF. Clinical characteristics, including comorbidities, were obtained from medical records. Congestive heart failure was defined as dyspnea associated with clinical signs of pulmonary edema and was confirmed by consistent findings on chest X-ray.

The study protocol was approved by the Institution Research and Ethics Committee of the University of Toyama (Toyama, Japan) and was conducted in accordance with the principles of the Declaration of Helsinki. Furthermore, we obtained written informed consent from patients before performing AF ablation.

\section{Measurement of Hemodynamic Parameters}

We measured SV using ICG (Aesculon ${ }^{\circledR}$ mini; OSYPKA Medical, Berlin, Germany) immediately before AF ablation, on the day following ablation, and 1,3, and 6 months after ablation. The SV immediately before ablation was measured while the patient was awake. Before ablation, SV was measured during sinus rhythm and AF rhythm in patients with paroxysmal and persistent AF, respectively. After ablation, SV was measured during sinus rhythm in all patients. All measurements of SV were performed after the patient rested for $>5 \mathrm{~min}$ in a supine position until both the heart rate and SV were stable. Briefly, 2 pairs of surface electrodes located side by side in a vertical direction were applied to the left side of the neck and the left lower thorax at the level of the xiphoid process. The electrodes were then connected to the ICG, which interpreted the maximum rate of change in thoracic bioimpedance as the ohmic equivalent of the mean aortic flow acceleration. In addition, the conductivity change because of the change in the blood conductivity was extracted to evaluate SV according to the Bernstein-Osypka equation. ${ }^{9}$ Furthermore, the SV index (SVI) was evaluated by dividing the SV by the body surface area, which was determined using the formula of Du Bois. ${ }^{17}$ The normal range of the SVI we used was $33-47 \mathrm{~mL} / \mathrm{m}^{2} .^{18}$

In addition, we measured the SVI using the thermodilu- tion method as a reference in 35 patients, including 20 patients with sinus rhythm and 15 patients with AF rhythm, immediately before ablation, in an awake state. A correlation between the SVI measured using ICG and that measured using thermodilution was analyzed to assess the validity of the SVI measured using ICG.

\section{Laboratory and Echocardiographic Data}

We performed laboratory tests and transthoracic echocardiography before and 6 months after AF ablation. Echocardiographic findings were assessed by an experienced cardiac sonographer blinded to other study findings. In addition, we measured the left atrial dimension (LAD), LV end-diastolic dimension (LVDd), and LV endsystolic dimension from the parasternal long-axis M-mode recordings. Using the Teichholz method, we evaluated the LVEF. Transesophageal echocardiography was performed with patients under sedation using diazepam within 3 days before ablation. Of note, AF ablation was cancelled if a left atrial thrombus was observed. We measured also the left atrial appendage flow velocity during the examination.

\section{AF Ablation}

All antiarrhythmic drugs were discontinued for at least 5 half-lives, and no patients received oral amiodarone before ablation. We used either the NavX System (St. Jude Medical Inc., St. Paul, MN, USA) or the CARTO System (Biosense-Webster, Inc., Diamond Bar, CA, USA) for 3D mapping. Sheath introducers were inserted through the right femoral vein of patients under sedation. In addition, the trans-septal procedure was performed, and three $8 \mathrm{~F}$ SL0 sheaths (St. Jude Medical Inc.) or two 8F SL0 sheaths and a steerable sheath (Agilis; St. Jude Medical Inc.) were advanced into the left atrium (LA). Pulmonary vein isolation (PVI) was performed in all patients. Furthermore, additional radiofrequency application targeting the complex fractionated atrial electrogram $(\mathrm{CFAE} ; \mathrm{n}=17)$ or LA posterior wall isolation with mitral isthmus line ablation $(n=47)$ was performed if the AF did not terminate or was induced with atrial burst pacing following PVI.

For ablation targeting the CFAE, we performed fractionation analyses on the NavX system. The mapping parameter (CFAE-mean) was defined as an interval-analysis algorithm that evaluated the average index of the fractionation. Moreover, a continuous CFAE was defined by an average fractionated index $\leq 50 \mathrm{~ms}$, suggesting the high temporal stability of the fractionated electrograms maintaining AF. ${ }^{19}$ We targeted all continuous CFAE sites for ablation.

For LA posterior wall isolation, we created the LA roof line at the most cranial aspect of the LA roof and a floor line joining the most inferior margin of the PVI line for LA posterior wall isolation. If the LA posterior wall was not isolated, we performed additional radiofrequency applications that targeted the earliest activation site on the isolation lines. Although the entrance block was confirmed by voltage mapping using a 3D mapping system, the exit block was confirmed using high-output pacing within the LA posterior wall isolation line. If AF continued despite the additional ablation procedure, external cardioversion was performed. The procedure was completed after cavotricuspid isthmus ablation. Of note, each radiofrequency application was performed for 30-50 s. The temperature was maintained at $42^{\circ} \mathrm{C}$ and power at $30 \mathrm{~W}$; the maximum power of $25 \mathrm{~W}$ was used while delivering energy to sites near the esophagus. 


\begin{tabular}{|c|c|c|c|c|}
\hline & $\begin{array}{c}\text { All patients } \\
(n=178)\end{array}$ & $\begin{array}{l}\text { Paroxysmal AF } \\
\text { patients }(n=108)\end{array}$ & $\begin{array}{l}\text { Persistent AF } \\
\text { patients }(n=70)\end{array}$ & $P$ value \\
\hline Age, years & $64 \pm 11$ & $65 \pm 11$ & $63 \pm 10$ & 0.267 \\
\hline Male sex & $131(74)$ & $74(69)$ & $57(81)$ & 0.083 \\
\hline AF duration, months & $23 \pm 24$ & - & $23 \pm 24$ & - \\
\hline Congestive heart failure & $19(11)$ & $6(6)$ & $13(19)$ & 0.013 \\
\hline Hypertension & $92(52)$ & $57(53)$ & $35(50)$ & 0.835 \\
\hline Diabetes mellitus & $22(12)$ & $15(14)$ & $7(10)$ & 0.591 \\
\hline Past history of stroke & $17(10)$ & $10(9)$ & $7(10)$ & $>0.999$ \\
\hline Antiarrhythmic drugs before ablation & $63(35)$ & $49(45)$ & $14(20)$ & 0.001 \\
\hline Antiarrhythmic drugs after ablation & $56(31)$ & $12(11)$ & $44(63)$ & $<0.001$ \\
\hline$\beta$-blockers before ablation & $87(49)$ & $49(45)$ & $38(54)$ & 0.313 \\
\hline$\beta$-blockers after ablation & $70(39)$ & $38(35)$ & $32(46)$ & 0.212 \\
\hline Calcium-channel blockers before ablation & $17(10)$ & $9(8)$ & $8(11)$ & 0.671 \\
\hline Calcium-channel blockers after ablation & $6(3)$ & $4(4)$ & $2(3)$ & $>0.999$ \\
\hline Left atrial appendage flow velocity, $\mathrm{cm} / \mathrm{s}$ & $56 \pm 29$ & $69 \pm 26$ & $35 \pm 21$ & $<0.001$ \\
\hline Left atrial dimension, $\mathrm{mm}$ & $41 \pm 6$ & $39 \pm 6$ & $44 \pm 6$ & $<0.001$ \\
\hline LV end-diastolic dimension, $\mathrm{mm}$ & $47 \pm 5$ & $48 \pm 5$ & $46 \pm 6$ & 0.047 \\
\hline LV ejection fraction, \% & $64 \pm 8$ & $65 \pm 7$ & $63 \pm 9$ & 0.272 \\
\hline $\mathrm{BNP}, \mathrm{pg} / \mathrm{mL}$ & $97 \pm 124$ & $51 \pm 54$ & $168 \pm 163$ & $<0.001$ \\
\hline eGFR, mL/min/1.73 $\mathrm{m}^{2}$ & $66 \pm 16$ & $66 \pm 17$ & $66 \pm 14$ & 0.880 \\
\hline Heart rate, beats/min & $74 \pm 18$ & $68 \pm 15$ & $83 \pm 18$ & $<0.001$ \\
\hline Stroke volume index, $\mathrm{mL} / \mathrm{m}^{2}$ & $33 \pm 6$ & $35 \pm 6$ & $30 \pm 5$ & $<0.001$ \\
\hline Reduced stroke volume index & $98(55)$ & $42(39)$ & $56(80)$ & $<0.001$ \\
\hline
\end{tabular}

Data are mean \pm SD or number (\%) of patients. AF, atrial fibrillation; BNP, B-type natriuretic peptide; eGFR, estimated glomerular filtration rate; $L V$, left ventricular.

\section{Postprocedural Care and Follow-up}

We conducted a clinical interview and performed a surface ECG on the day following ablation and at monthly visits to the outpatient clinic after that. In addition, we performed 24-h Holter monitoring during the follow-up period. Antiarrhythmic drugs were administered at the discretion of the treating physician.

\section{Statistical Analysis}

We assessed changes in the time course of the hemodynamic, echocardiographic, and laboratory parameters separately for patients with paroxysmal and persistent AF. In addition, these parameters were compared between patients with preserved SVI (preserved SVI group, SVI $\geq 33 \mathrm{~mL} / \mathrm{m}^{2}$ ) and those with reduced SVI (reduced SVI group, SVI $<33 \mathrm{~mL} / \mathrm{m}^{2}$ ). We defined cardiac function improvement as a $20 \%$ increase in SVI at 6 months after ablation and assessed the predictors of cardiac function improvement after AF ablation.

In this study, data are presented as mean \pm standard deviation with $95 \%$ confidence intervals. We analyzed the significance of differences between groups using the unpaired Student's t-test for continuous variables and the $X^{2}$ test for categorical variables. In addition, the time course of changes in the hemodynamic parameters was analyzed using two-way repeated measures analysis of variance. If significant changes were observed, posthoc tests with Bonferroni-adjusted pairwise comparisons were performed. We analyzed the time course of changes in other parameters using the paired Student's t-test. The correlation was tested using Pearson's correlation coefficient. Furthermore, we performed a multiple logistic regression analysis to assess variables related to cardiac function improvement following ablation. In this model, we adjusted for variables with significant differences in the univariate analysis. Moreover, receiver-operating characteristic curve analyses were performed to evaluate the optimal cutoff values for estimating cardiac function improvement following ablation. We considered $\mathrm{P}<0.05$ as statistically significant.

\section{Results}

\section{Baseline Patient Characteristics}

The mean age of the study population was $64 \pm 11$ years, and $74 \%$ of patients were male (Table 1). Congestive heart failure was present in $11 \%$ of the study population. Although the mean LVEF was in the normal range, we observed a reduced SVI in $55 \%$ of patients. Mean AF duration of patients with persistent $\mathrm{AF}$ was $23 \pm 24$ months, and 37 patients $(52 \%)$ had longstanding persistent AF. In this study, the prevalence of congestive heart failure was higher in patients with persistent AF than in those with paroxysmal AF. In addition, the rate of administration of antiarrhythmic drugs was lower in patients with persistent AF than in those with paroxysmal AF before ablation; however, it was higher in patients with persistent AF than in those with paroxysmal AF after ablation. The LA appendage flow velocity was lower, and the LAD was greater in patients with persistent $\mathrm{AF}$ than in those with paroxysmal AF. The B-type natriuretic peptide (BNP) level of patients with persistent $\mathrm{AF}$ was higher than that of patients with paroxysmal AF. The heart rate was higher in patients with persistent AF than in those with paroxysmal AF. The SVI was lower in patients with persistent AF than in those with paroxysmal AF. Furthermore, a reduced SVI was observed in $39 \%$ and $80 \%$ of patients with paroxysmal 


\section{A. Sinus rhythm}

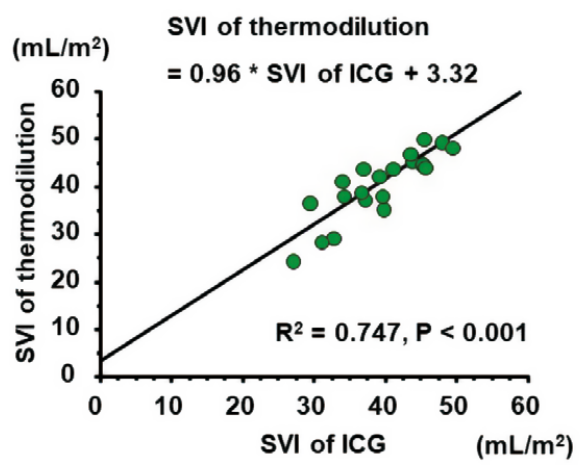

\section{B. AF rhythm}

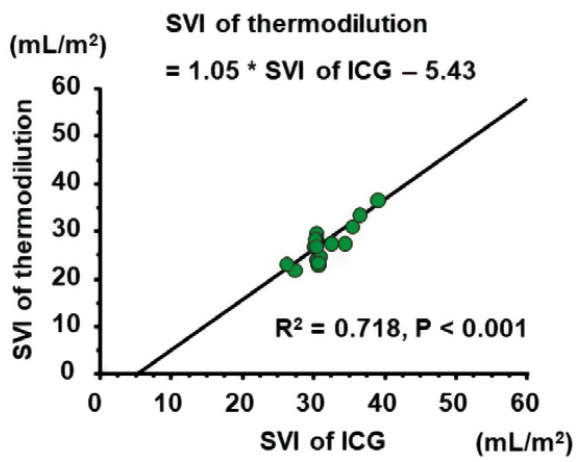

Figure 1. Correlation between the stroke volume index (SVI) measured using impedance cardiography (ICG) and that measured using the thermodilution method during sinus rhythm $(\mathbf{A})$ and atrial fibrillation $(\mathrm{AF})$ rhythm (B).
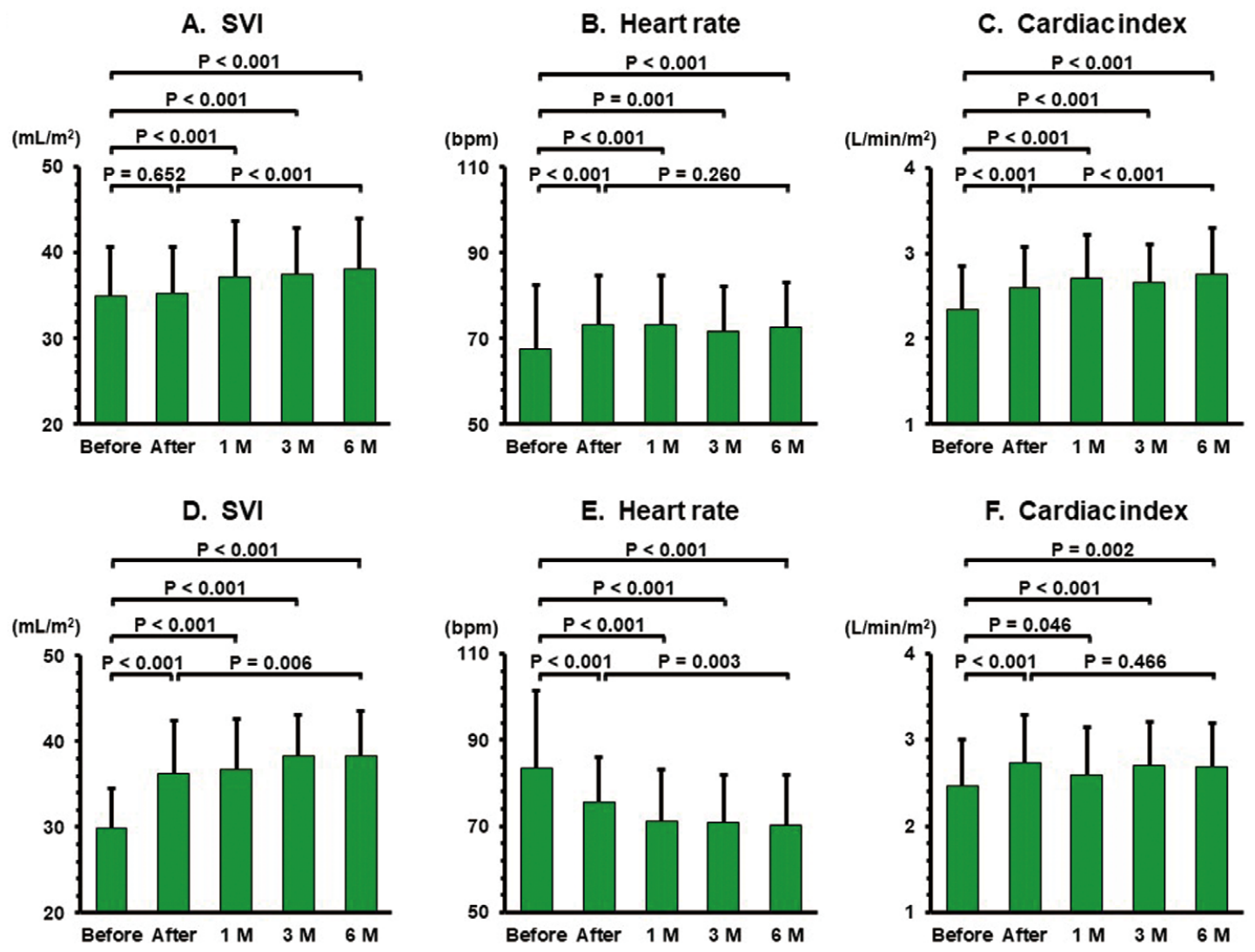

Figure 2. Time course of changes in hemodynamic parameters. The stroke volume index (SVI, A), heart rate (B), and cardiac index $(\mathbf{C})$ in patients with paroxysmal AF, as well as the SVI (D), heart rate $(\mathbf{E})$, and cardiac index $(\mathbf{F})$ in patients with persistent $\mathrm{AF}$, are shown. M, month/months.

and persistent $\mathrm{AF}$, respectively.

\section{SVI Measured Using ICG and the Thermodilution Method}

The SVI measured using ICG significantly correlated with that measured using thermodilution during both sinus rhythm $\left(\mathrm{R}^{2}=0.747 ; \mathrm{P}<0.001\right.$; Figure 1A) and AF rhythm $\left(\mathrm{R}^{2}=0.718 ; \mathrm{P}<0.001\right.$; Figure 1B). As an intercept of the regression line was positive during sinus rhythm (Figure 1A), the SVI measured using ICG was smaller than that measured using thermodilution. In contrast, as an intercept of 

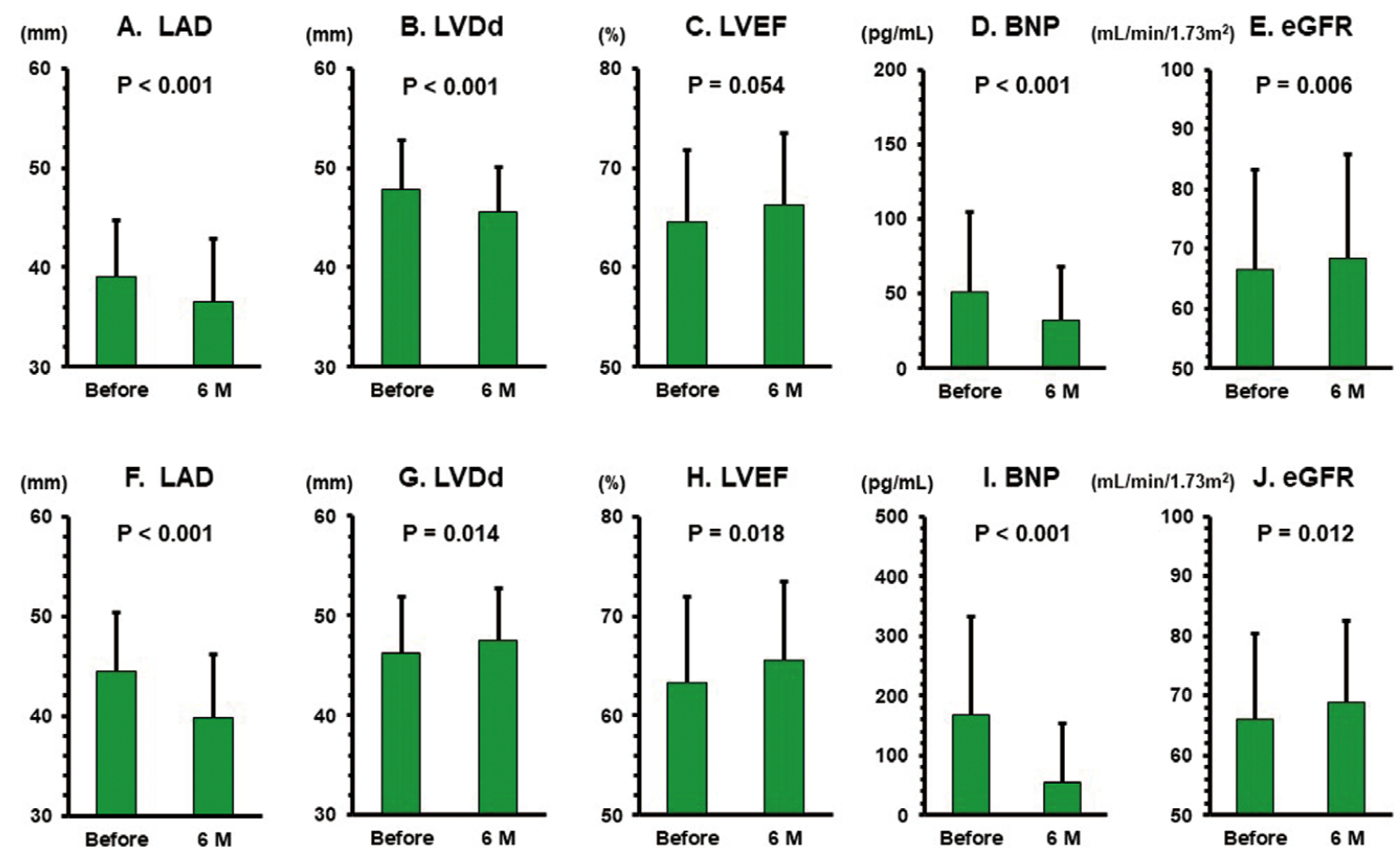

Figure 3. Time course of changes in echocardiographic and laboratory data. The left atrial dimension (LAD, A), left ventricular end-diastolic dimension (LVDd, B), left ventricular ejection fraction (LVEF, C), B-type natriuretic peptide (BNP, D) level, and estimated glomerular filtration rate (eGFR, E) in patients with paroxysmal AF, as well as the LAD (F), LVDd (G), LVEF (H), BNP (I), and eGFR $(\mathbf{J})$ in patients with persistent $A F$, are shown. M, months.

the regression line was negative during $\mathrm{AF}$ rhythm (Figure 1B), the SVI measured using ICG was larger than that measured using thermodilution during AF rhythm.

\section{Time Course of Changes in Hemodynamic Parameters}

In patients with paroxysmal AF, the SVI did not change immediately following ablation (from $35 \pm 6 \mathrm{~mL} / \mathrm{m}^{2}$ to $35 \pm 5 \mathrm{~mL} / \mathrm{m}^{2} ; \mathrm{P}=0.652$; Figure $2 \mathrm{~A}$ ); however, a gradual increase was observed, and the degree of change attained statistical significance at 1 month after ablation (1 month, $37 \pm 6 \mathrm{~mL} / \mathrm{m}^{2}, \mathrm{P}<0.001$ vs. before ablation; 3 months, $37 \pm 5 \mathrm{~mL} / \mathrm{m}^{2}, \mathrm{P}<0.001 ; 6$ months, $38 \pm 6 \mathrm{~mL} / \mathrm{m}^{2}, \mathrm{P}<0.001$ ). In addition, the heart rate increased immediately after ablation and subsequently remained unchanged (Figure 2B). Consequently, the heart rate at 6 months after ablation was higher than that before ablation. Together with the increase in the SVI and heart rate, the cardiac index (CI) gradually increased after ablation (Figure 2C).

In patients with persistent $\mathrm{AF}$, the SVI increased immediately after ablation (from $30 \pm 5 \mathrm{~mL} / \mathrm{m}^{2}$ to $36 \pm 6 \mathrm{~mL} / \mathrm{m}^{2}$; $\mathrm{P}<0.001$; Figure 2D) and further increased after that (1 month, $37 \pm 6 \mathrm{~mL}, \mathrm{P}<0.001$ vs. before ablation; 3 months, $38 \pm 5 \mathrm{~mL} / \mathrm{m}^{2}, \mathrm{P}<0.001 ; 6$ months, $38 \pm 5 \mathrm{~mL} / \mathrm{m}^{2}, \mathrm{P}<0.001$ ). However, the heart rate decreased immediately after ablation and further decreased after that (Figure 2E). Furthermore, the CI markedly increased after ablation because the extent of increase in the SVI was higher than the extent of reduction in the heart rate (Figure 2F).

\section{Changes in Echocardiographic and Laboratory Data}

The LAD decreased 6 months after ablation in both patients with paroxysmal and persistent AF (Figure 3A,F). The LVDd became smaller in patients with paroxysmal AF (Figure 3B); however, it became larger in patients with persistent AF (Figure 3G). In addition, the LVEF tended to increase in patients with paroxysmal AF (Figure 3C) and significantly increased in patients with persistent AF (Figure 3H). Furthermore, the BNP level reduced (Figure 3D,I), but the estimated glomerular filtration rate (eGFR) increased in both patient groups (Figure 3E,J).

\section{Comparison of Hemodynamic Parameters in the Preserved and Reduced SVI Groups}

In patients with paroxysmal AF, the SVI tended to increase in the preserved SVI group 6 months after ablation and significantly increased in the reduced SVI group (Figure 4A). The degree of increase in the SVI was smaller in the preserved SVI group than in the reduced SVI group $\left(1 \pm 6 \mathrm{~mL} / \mathrm{m}^{2}\right.$ vs. $\left.6 \pm 5 \mathrm{~mL} / \mathrm{m}^{2} ; \mathrm{P}<0.001\right)$. In addition, the SVI was higher in the preserved SVI group than in the reduced SVI group throughout the follow-up period. Although the heart rate increased in the preserved SVI group, it remained unchanged in the reduced SVI group (Figure 4B). In addition, the heart rate was lower in the preserved SVI group than in the reduced SVI group before ablation; however, it was not different between the groups after ablation. The CI markedly increased in both the preserved and reduced SVI groups (Figure 4C). As the degree of increase in the CI was smaller in the preserved SVI group than in the reduced SVI 


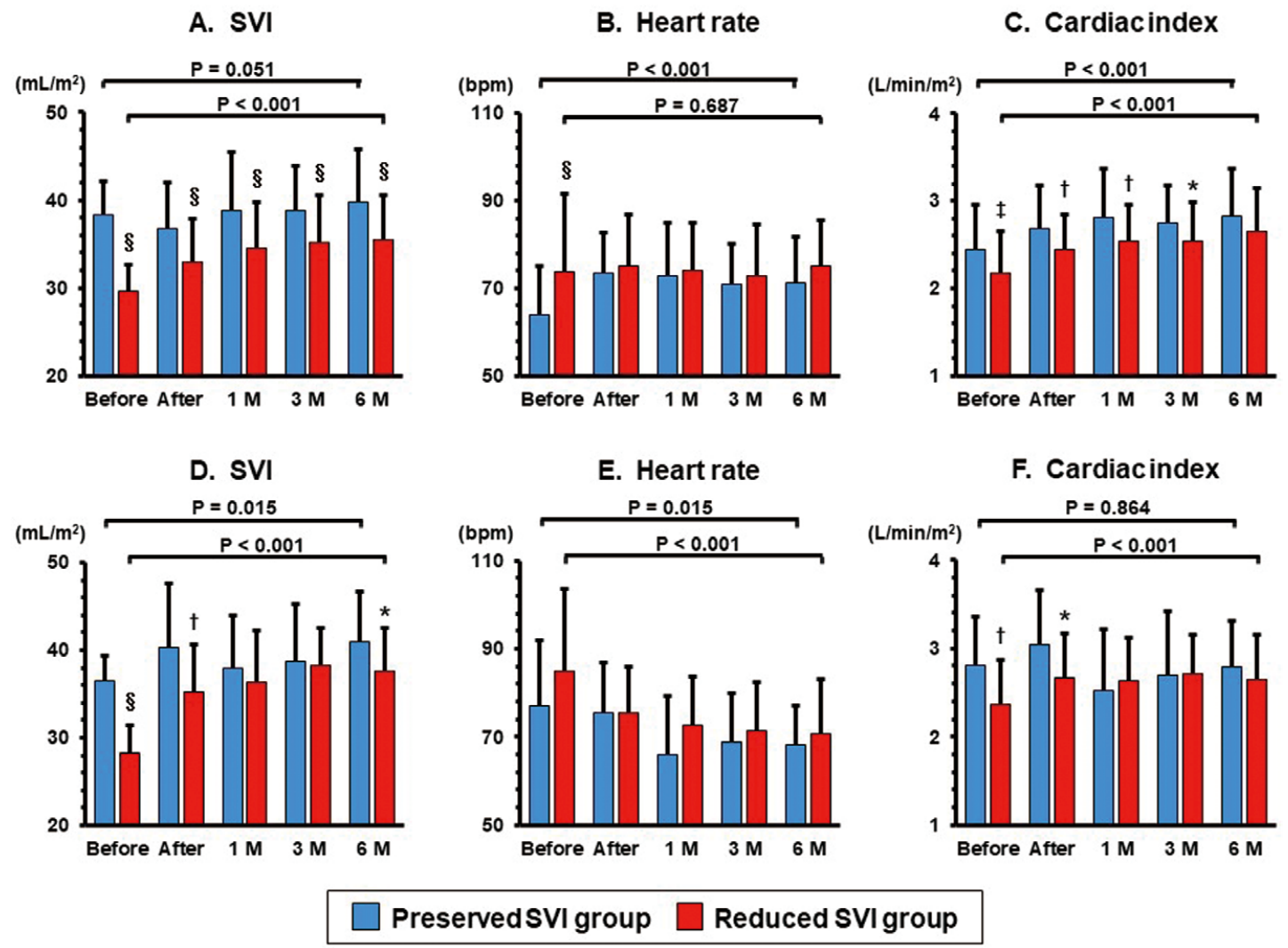

Figure 4. Comparison of changes in the time course of hemodynamic parameters between the preserved (blue bars) and reduced (red bars) stroke volume index (SVI) groups. The SVI (A), heart rate (B), and cardiac index (C) in patients with paroxysmal AF, as well as the SVI (D), heart rate $(\mathbf{E})$, and cardiac index $(\mathbf{F})$ in patients with persistent AF, are shown. M, month or months.

group, the difference in the CI between the groups lost statistical significance 6 months after ablation.

In patients with persistent AF, the SVI increased in both the preserved and reduced SVI groups (Figure 4D); however, the degree of increase in the SVI was smaller in the preserved SVI group than in the reduced SVI group $\left(4 \pm 6 \mathrm{~mL} / \mathrm{m}^{2}\right.$ vs. $\left.9 \pm 5 \mathrm{~mL} / \mathrm{m}^{2} ; \mathrm{P}=0.003\right)$. The SVI was still higher in the preserved SVI group than in the reduced SVI group 6 months after ablation. The heart rate gradually decreased after ablation in both the preserved and reduced SVI groups and was not different between the groups throughout the follow-up period (Figure 4E). The CI did not change in the preserved SVI group (Figure 4F); however, it increased in the reduced SVI group. Consequently, the difference in the CI between the groups disappeared 1 month after ablation.

\section{Predictors of Improvement in Cardiac Function}

We observed an improvement in cardiac function in 70 (39\%) patients, including $30(28 \%)$ and $40(57 \%)$ patients with paroxysmal and persistent AF, respectively. Patients with improved cardiac function were younger than those without any such improvement (Table 2). In addition, the proportion of patients with persistent AF was higher, and the administration rate of antiarrhythmic drugs before ablation was lower in patients with improved cardiac function compared with those without. Furthermore, the
LA appendage flow velocity, LVEF, and baseline SVI were lower, and the baseline heart rate and proportion of patients with reduced baseline SVI were higher in patients with improved cardiac function compared with those without.

After adjustment for age, AF type, the administration rate of antiarrhythmic drugs before ablation, LA appendage flow velocity, LVEF, baseline heart rate, and baseline SVI, the multivariate analysis revealed that age and baseline SVI correlated with improved cardiac function after ablation (Table 3). In addition, age attained an area under the curve of 0.653 for the ability to predict improvement in cardiac function; the sensitivity and specificity were $57 \%$ and $71 \%$, respectively, for a cutoff value of 64 years. Furthermore, the baseline SVI attained an area under the curve of 0.828 ; the sensitivity and specificity were $77 \%$ and $79 \%$, respectively, for a cutoff value of $32 \mathrm{~mL} / \mathrm{m}^{2}$.

\section{Discussion}

This study assessed hemodynamic parameters using ICG and determined that a potential reduction in the SVI existed in $55 \%$ of patients with AF (paroxysmal AF, 39\%; persistent $\mathrm{AF}, 80 \%$ ) and preserved LV systolic function. After ablation, the SVI and CI increased in patients with paroxysmal and persistent AF. In addition, the LAD decreased, LVEF increased, and BNP decreased following 


\begin{tabular}{|c|c|c|c|}
\hline & $\begin{array}{l}\text { Improvement in cardiac } \\
\text { function }(n=70)\end{array}$ & $\begin{array}{l}\text { No improvement in } \\
\text { cardiac function }(n=108)\end{array}$ & $P$ value \\
\hline Age, years & $61 \pm 11$ & $66 \pm 10$ & $<0.001$ \\
\hline Male sex & $52(74)$ & $79(73)$ & $>0.999$ \\
\hline AF type, persistent $\mathrm{AF}$ & $40(57)$ & $30(28)$ & $<0.001$ \\
\hline AF duration, months & $23 \pm 28$ & $22 \pm 17$ & 0.857 \\
\hline Congestive heart failure & $9(13)$ & $10(9)$ & 0.609 \\
\hline Hypertension & $34(49)$ & $58(54)$ & 0.606 \\
\hline Diabetes mellitus & $10(14)$ & $12(11)$ & 0.693 \\
\hline Past history of stroke & $8(11)$ & $9(8)$ & 0.671 \\
\hline Antiarrhythmic drugs before ablation & $17(24)$ & $46(43)$ & 0.020 \\
\hline Antiarrhythmic drugs after ablation & $27(39)$ & $29(27)$ & 0.139 \\
\hline$\beta$-blockers before ablation & $39(56)$ & $48(44)$ & 0.188 \\
\hline$\beta$-blockers after ablation & $31(44)$ & $39(36)$ & 0.351 \\
\hline Calcium-channel blockers before ablation & $5(7)$ & $12(36)$ & 0.536 \\
\hline Calcium-channel blockers after ablation & $2(3)$ & $4(4)$ & $>0.999$ \\
\hline Left atrial appendage flow velocity, $\mathrm{cm} / \mathrm{s}$ & $46 \pm 26$ & $62 \pm 30$ & $<0.001$ \\
\hline Left atrial dimension, $\mathrm{mm}$ & $42 \pm 6$ & $41 \pm 7$ & 0.207 \\
\hline LV end-diastolic dimension, $\mathrm{mm}$ & $47 \pm 5$ & $47 \pm 5$ & 0.411 \\
\hline LV ejection fraction, \% & $62 \pm 8$ & $65 \pm 8$ & 0.015 \\
\hline BNP, pg/mL & $117 \pm 125$ & $84 \pm 123$ & 0.094 \\
\hline eGFR, $\mathrm{mL} / \mathrm{min} / 1.73 \mathrm{~m}^{2}$ & $68 \pm 15$ & $66 \pm 16$ & 0.416 \\
\hline Heart rate before ablation, beats/min & $81 \pm 21$ & $69 \pm 14$ & $<0.001$ \\
\hline Heart rate 6 months after ablation, beats/min & $71 \pm 11$ & $72 \pm 11$ & 0.552 \\
\hline Stroke volume index, $\mathrm{mL} / \mathrm{m}^{2}$ & $29 \pm 4$ & $35 \pm 5$ & $<0.001$ \\
\hline Reduced stroke volume index & $59(84)$ & $39(36)$ & $<0.001$ \\
\hline
\end{tabular}

Data are mean \pm SD or number (\%) of patients. Abbreviations as in Table 1.

\begin{tabular}{|lcc|}
\hline Table 3. Multivariate Analysis for Predictors of Improvement in Cardiac Function Following AF Ablation \\
& $\begin{array}{c}\text { OR } \\
\text { (95\% confidence interval) }\end{array}$ & P value \\
Age, years & $1.090(1.042-1.141)$ & $<0.001$ \\
AF type, persistent AF & $1.396(0.481-4.051)$ & 0.540 \\
Antiarrhythmic drugs before ablation & $1.030(0.415-2.556)$ & 0.949 \\
Left atrial appendage flow velocity, cm/s & $1.016(0.999-1.033)$ & 0.071 \\
LV ejection fraction, \% & $0.999(0.947-1.054)$ & 0.980 \\
Heart rate before ablation, beats/min & $1.004(0.977-1.031)$ & 0.783 \\
Stroke volume index, $\mathrm{mL} / \mathrm{m}^{2}$ & $1.367(1.209-1.545)$ & $<0.001$ \\
\hline
\end{tabular}

OR, odds ratio. Other abbreviations as in Table 1.

ablation. Furthermore, the degree of increment in the SVI was higher in patients with a reduced baseline SVI than in those with a preserved baseline SVI, and the baseline SVI was the most reliable predictor of an improvement in cardiac function after ablation.

\section{AF Ablation and Cardiac Function}

The appropriate assessment of cardiac function is challenging in patients with AF because both atrial and ventricular function is impaired in these patients. Typically, the LVEF is used for assessing cardiac function in patients with $\mathrm{AF},{ }^{\mathbf{4}}$ - but it does not entirely reflect the atrial function. Prior studies have used exercise capacity ${ }^{4}$ and peak oxygen consumption $^{20}$ as indicators of AF ablation efficacy; however, these parameters are affected by multiple factors, including respiratory function and muscle mass, and, therefore, might not necessarily represent total cardiac function. In contrast, SV directly represents global cardiac function, including both atrial and ventricular function. Consequently, we observed a potential reduction in SV in over half of the patients with AF and preserved LV systolic function. Furthermore, we observed a reduction in SV in $80 \%$ of patients with persistent AF. These findings suggested a marked effect of atrial contractile dysfunction on global cardiac function in patients with AF.

Unlike in a previous study, ${ }^{7}$ we observed an improvement in cardiac function of patients with AF and preserved LV systolic function. In this study, an improvement in global cardiac function might have been correctly assessed. Notably, the degree of increase in SV was especially high in patients with a reduced baseline SV. The effect of AF on cardiac function might have been substantial in these patients. Thus, AF ablation should be considered for improving cardiac function in patients with a reduced $\mathrm{SV}$, even if they 
have normal LV systolic function. A recent randomized trial reported that AF ablation in patients with LV systolic dysfunction correlated with a better outcome compared with medical therapy. ${ }^{21}$ Furthermore, AF ablation in patients with a potential reduction in SV might also contribute to improvement in the clinical outcome.

In this study, the time course of increase in SV was different between patients with paroxysmal and persistent $\mathrm{AF}$. There was no immediate rise in SV after ablation in patients with paroxysmal AF because the measurement of SV before ablation was performed during sinus rhythm in all patients with paroxysmal AF. However, SV increased immediately after ablation in patients with persistent AF. The restoration of sinus rhythm and atrial contraction should have contributed to an immediate increase in SV in patients with persistent AF. Notably, SV increased during the follow-up period in patients with paroxysmal and persistent AF. Gradual recovery from atrial contractile dysfunction and tachycardia-induced cardiac dysfunction might have contributed to the increase in SV. ${ }^{14,15}$

The heart rate of patients with paroxysmal AF increased after ablation. Reportedly, vagal nerve modification during PVI can contribute to this increase in the heart rate. ${ }^{22}$ In contrast, the heart rate decreased after ablation in patients with persistent AF because of sinus restoration. Notably, cardiac output gradually increased after ablation in both patient groups, regardless of the alteration in heart rate. Furthermore, although the baseline heart rate was higher in patients with improved cardiac function than in those without, the increase in SV was independent of the baseline heart rate. Heart rate is regulated by autonomic nerves according to the cardiac output required by the body tissues; thus, the heart rate might have decreased because the demand on cardiac output had been satisfied by the increased SV. This hypothesis is supported by the improved renal function after ablation. The increase in cardiac output could have contributed to increased renal blood flow and consequent improvement in renal function.

The LVEF increased 6 months after ablation, despite LV systolic function being in the normal range before ablation, suggesting that mild tachycardia-induced cardiac dysfunction might exist in patients with AF and no apparent LV systolic dysfunction. In addition, we could successfully control the heart rate using drugs; thus, tachycardia was not observed before ablation. However, the heart rate was measured during sinus rhythm in most patients with paroxysmal AF before ablation; thus, tachycardia might have occurred during AF in patients with paroxysmal AF. Furthermore, an increase in the heart rate during exercise could have caused tachycardia-induced cardiac dysfunction in patients with persistent AF. These findings are consistent with those reported previously, ${ }^{4}$ wherein the LVEF increased, even in patients with appropriate rate control.

The LVDd enlarged after ablation in patients with persistent AF. The mechanism of the enlargement of LVDd after ablation is unclear; however, the shortening of the diastolic phase because of tachycardia may have caused the reduction in LVDd before ablation.

\section{Validity of SV Measured Using ICG}

Studies have established the validity of ICG for measuring SV during sinus rhythm. ${ }^{\mathbf{8 1 0 - 1 2}}$ However, the measurement of SV might be more challenging during AF rhythm than sinus rhythm. A study reported that SV measured using ICG was more accurate than that measured using the inert gas rebreathing method. ${ }^{13}$ Likewise, the SV measured using ICG markedly correlated with that measured using the thermodilution method in this study during both sinus rhythm and AF rhythm. Thus, the measurement of SV using ICG is valid during both sinus rhythm and AF rhythm. Another study reported that the intraindividual variability was smaller than the interindividual variability in the ICG measurement because the influence of body size and shape can be excluded. ${ }^{12}$ In the present study, intraindividual changes in ICG parameters were evaluated, so the results presented seem reliable.

In this study, the SV measured using ICG was smaller than the reference value obtained during sinus rhythm but was higher than the reference during AF rhythm. If these findings are accommodated, the degree of increase in SV immediately after ablation in patients with persistent AF becomes higher than that indicated in Figure 2D.

\section{Study Limitations}

First, the measurement conditions might have affected hemodynamic parameters. However, ICG was performed after adequate rest time, and the value reaching the plateau was adopted. Second, antiarrhythmic drugs, calcium-channel blockers, and $\beta$-blockers might have affected cardiac function, all such drugs were discontinued before the measurement of SV at baseline, and the administration rate of antiarrhythmic drugs after ablation was not different between patients with and those without improved cardiac function. The study protocol did not consider the discontinuation of calcium-channel blockers and $\beta$-blockers. However, the effect of these drugs might have been weak because the administration rate of these drugs was similar between patients with and without improvement in cardiac function. Third, patients with AF recurrence were excluded, so the effect of AF ablation on cardiac function in patients with AF recurrence remains unclear. Finally, because atrial contractile function was not directly assessed, we could not conclude that recovery of the atrial contractile function contributed to the increase in SV. However, the LAD became smaller after ablation, and the increase in SV was independent of baseline LVEF. These findings suggested the contribution of the atrial reverse remodeling on the increase in SV.

\section{Conclusions}

This study revealed a potential reduction in SV in over half of the study cohort comprising patients with AF and preserved LV systolic function. In addition, the study suggested that restoration and maintenance of sinus rhythm using catheter ablation improved cardiac function in patients with paroxysmal or persistent $\mathrm{AF}$, and the degree of improvement in cardiac function is high in patients with reduced SV.

\section{Acknowledgments}

This research received no grant from any funding agency in the public, commercial or not-for-profit sectors.

\section{References}

1. Benjamin EJ, Wolf PA, D'Agostino RB, Silbershatz H, Kannel WB, Levy D. Impact of atrial fibrillation on the risk of death: The Framingham Heart Study. Circulation 1998; 98: 946-952.

2. Santhanakrishnan R, Wang N, Larson MG, Magnani JW, McManus DD, Lubitz SA, et al. Atrial fibrillation begets heart failure and vice versa: Temporal associations and differences in 
preserved versus reduced ejection fraction. Circulation 2016; 133: 484-492.

3. Gómez-Outes A, Lagunar-Ruíz J, Terleira-Fernández AI, CalvoRojas G, Suárez-Gea ML, Vargas-Castrillón E. Causes of death in anticoagulated patients with atrial fibrillation. $\mathrm{J} \mathrm{Am} \mathrm{Coll}$ Cardiol 2016; 68: 2508-2521.

4. Hsu LF, Jaïs P, Sanders P, Garrigue S, Hocini M, Sacher F, et al. Catheter ablation for atrial fibrillation in congestive heart failure. N Engl J Med 2004; 351: 2373-2383.

5. Anselmino M, Matta M, D'Ascenzo F, Bunch TJ, Schilling RJ, Hunter RJ, et al. Catheter ablation of atrial fibrillation in patients with left ventricular systolic dysfunction: A systematic review and meta-analysis. Circ Arrhythm Electrophysiol 2014; 7: $1011-1018$.

6. Ling LH, Taylor AJ, Ellims AH, Iles LM, McLellan AJ, Lee G, et al. Sinus rhythm restores ventricular function in patients with cardiomyopathy and no late gadolinium enhancement on cardiac magnetic resonance imaging who undergo catheter ablation for atrial fibrillation. Heart Rhythm 2013; 10: 1334-1339.

7. Lutomsky BA, Rostock T, Koops A, Steven D, Müllerleile K, Servatius H, et al. Catheter ablation of paroxysmal atrial fibrillation improves cardiac function: A prospective study on the impact of atrial fibrillation ablation on left ventricular function assessed by magnetic resonance imaging. Europace 2008; 10: 593-599.

8. Cybulski G, Strasz A, Niewiadomski W, Gąsiorowska A. Impedance cardiography: Recent advancements. Cardiol J 2012; 19: $550-556$.

9. Bernstein DP, Lemmens HJ. Stroke volume equation for impedance cardiography. Med Biol Eng Comput 2005; 43: $443-$ 450.

10. Rozenman Y, Rotzak R, Patterson RP. Detection of left ventricular systolic dysfunction using a newly developed, laptop based, impedance cardiographic index. Int J Cardiol 2011; 149: $248-250$.

11. Dupuis M, Noel-Savina E, Prévot G, Tétu L, Pillard F, Rivière D, et al. Determination of cardiac output in pulmonary hypertension using impedance cardiography. Respiration, doi:10.1159/000486423.

12. Limper U, Gauger P, Beck LEJ. Upright cardiac output measurements in the transition to weightlessness during parabolic flights. Aviat Space Environ Med 2011; 82: 448-454.
13. Osbak PS, Henriksen JH, Kofoed KF, Jensen GB. Non-invasive measurements of cardiac output in atrial fibrillation: Inert gas rebreathing and impedance cardiography. Scand J Clin Lab Invest 2011; 71: 304-313.

14. Sun H, Gaspo R, Leblanc N, Nattel S. Cellular mechanisms of atrial contractile dysfunction caused by sustained atrial tachycardia. Circulation 1998; 98: 719-727.

15. Grogan M, Smith HC, Gersh BJ, Wood DL. Left ventricular dysfunction due to atrial fibrillation in patients initially believed to have idiopathic dilated cardiomyopathy. Am J Cardiol 1992; 69: $1570-1573$

16. Calkins H, Kuck KH, Cappato R, Brugada J, Camm AJ, Chen SA, et al. 2012 HRS/EHRA/ECAS expert consensus statement on catheter and surgical ablation of atrial fibrillation: Recommendations for patient selection, procedural techniques, patient management and follow-up, definitions, endpoints, and research trial design: A report of the Heart Rhythm Society (HRS) Task Force on Catheter and Surgical Ablation of Atrial Fibrillation. Heart Rhythm 2012; 9: 632-696.e21.

17. DuBois D, DuBois EF. A formula to estimate the approximate surface area if height and weight be known. Arch Intern Med 1916; 17: 863-871.

18. Chiam E, Weinberg L, Bailey M, McNicol L, Bellomo R. The haemodynamic effects of intravenous paracetamol (acetaminophen) in healthy volunteers: A double-blind, randomized, triple crossover trial. Br J Clin Pharmacol 2016; 81: 605-612.

19. Lin YJ, Tai CT, Kao T, Chang SL, Lo LW, Tuan TC, et al. Spatiotemporal organization of the left atrial substrate after circumferential pulmonary vein isolation of atrial fibrillation. Circ Arrhythm Electrophysiol 2009; 2: 233-241.

20. Hunter RJ, Berriman TJ, Diab I, Kamdar R, Richmond L, Baker $\mathrm{V}$, et al. A randomized controlled trial of catheter ablation versus medical treatment of atrial fibrillation in heart failure (the CAMTAF trial). Circ Arrhythm Electrophysiol 2014; 7: 31 - 38.

21. Marrouche NF, Brachmann J, Andresen D, Siebels J, Boersma L, Jordaens L, et al. Catheter ablation for atrial fibrillation with heart failure. N Engl J Med 2018; 378: 417-427.

22. Pappone C, Santinelli V, Manguso F, Vicedomini G, Gugliotta $\mathrm{F}$, Augello G, et al. Pulmonary vein denervation enhances longterm benefit after circumferential ablation for paroxysmal atrial fibrillation. Circulation 2004; 109: 327-334. 